\title{
VALIDATION OF DRIVING SIMULATION TO ASSESS ON-ROAD PERFORMANCE IN HUNTINGTON DISEASE
}

\author{
Hannes Devos ${ }^{1,2}$, Alice Nieuwboer ${ }^{1}$, Wim Vandenberghe ${ }^{1}$, Mark Tant ${ }^{3}$, Willy De \\ Weerdt ${ }^{1}$ \& Ergun $\mathrm{Uc}^{2,4}$ \\ ${ }^{1}$ University of Leuven, Leuven, Belgium \\ ${ }^{2}$ University of Iowa, Iowa City, Iowa, USA \\ ${ }^{3}$ Belgian Road Safety Institute, Brussels, Belgium \\ ${ }^{4}$ Veterans Affairs Medical Center, Iowa City, Iowa, USA \\ Email: Hannes.Devos@faber.kuleuven.be
}

\begin{abstract}
Summary: Driving simulators are increasingly used to assess the driving capabilities of persons with neurodegenerative conditions. However, few driving simulator evaluations have been validated against standardized on-road tests. The aim of this study was to investigate the concurrent validity of a comprehensive driving simulator evaluation in 29 persons with Huntington disease (HD). The Test Ride for Investigating Practical fitness to drive (TRIP) checklist was administered after a $15 \mathrm{~km}$ simulator drive and $20 \mathrm{~km}$ on-road drive. The total driving simulator TRIP score and each of its item scores were compared with the on-road TRIP scores using Spearman rho correlation statistics. We found significant correlations for 9 of the 12 items. Correlations ranged between 0.12 for the item gap distance at speed below $50 \mathrm{~km} / \mathrm{h}$ and 0.72 for the total TRIP score, indicating variable strength of the associations. Items assessing operational skills correlated better with on-road driving performance than tactical or higher-order visual items. The results indicate that a fixed-base, single screen driving simulator is a valid tool to assess on-road driving capabilities in persons with HD.
\end{abstract}

\section{INTRODUCTION}

Potentially impaired drivers with neurological conditions are often referred for a road test to determine their driving capabilities. On-road tests are acclaimed to be the standard of fitness to drive in neurological conditions (Devos et al., 2011), provided that the driving performance is evaluated by standardized checklists with established reliability and validity (Fox et al., 1998). However, a number of environmental factors limit the standardization and repeatability of onroad tests. On-road assessments typically last 20 to 60 minutes and may not capture the effect of fluctuating symptoms (e.g., sleepiness, fatigue). On-road driving performance depends on the car that is used (own car versus car of the driving assessment center), weather conditions (good versus poor visibility), area of testing (rural versus urban; familiar versus unfamiliar) and the period of testing (during or outside peak hours). Other limitations include a lack of age-normed data and a limited validity of on-road assessments in predicting crash involvement (Akinwuntan et al., 2012; Uc et al., 2011). Above all, there is a risk involved with road tests, both for the driver, instructor as well as other road users.

Driving simulation has the capability of interactively engaging the motor, visual, and cognitive skills needed for safe driving in a controlled setting (Akinwuntan et al., 2012). However, standardized scenarios with established validity are needed to apply driving simulation in clinical 
realm to establish driver fitness. Validity refers to the degree that a scale measures what it claims to measure. Concurrent validity is a type of construct validity in which the measure of interest (driving simulation) is compared to a gold standard (road test) at approximately the same time. Drivers without neurological conditions exhibit a similar pattern of driving behavior in the simulator and on the road (Mayhew et al., 2011). The concurrent validity of driving simulation in neurologically impaired drivers is less clear. Only 3 studies investigated the relationship between performance in a driving simulator and on a road test after stroke. Nouri and Tinson failed to find a significant agreement between the driving simulator evaluation and the on-road decision (kappa $=0.29$ ). Patomella et al could not establish a clear cut-off score to predict on-road performance with acceptable sensitivity. Conversely, Lundqvist et al found that a driving simulation predicted on-road performance with better accuracy than did performance on neuropsychological tests (85\% versus $73 \%$ ). A meta-analysis of the 3 abovementioned studies produced a moderate effect size of 0.71 (Devos et al., 2011). In Parkinson's disease (PD), a driving simulator evaluation accounted for $39 \%$ of the total variance of on-road performance, which was substantially lower than the variance explained in older drivers without PD $\left(\mathrm{R}^{2}=68 \%\right)$. Still, the authors concluded that driving simulation has the potential to be used for screening of unsafe drivers with PD (Lee et al., 2007). In previous work, we found that adding a driving simulator evaluation to a clinical screening battery increased the accuracy to predict fitness to drive from $90 \%$ to $97.5 \%$ (Devos et al., 2007).

Huntington disease (HD) is an inherited, neurodegenerative condition that is associated with motor, cognitive, and behavioral deficits that may greatly affect safe driving (Beglinger et al., 2012). Driving simulator evaluations may be particularly indicated in this patient group because of their increased risk of car crashes (Rebok et al., 1995). So far, no studies have investigated the concurrent validity of driving simulator evaluations against road tests in HD, although two studies have shown good discriminant ability of driving simulator performance between drivers with HD and healthy age- and gender- matched drivers (Devos et al., 2012; Rebok et al., 1995). The aim of this study was to investigate the concurrent validity of a driving simulator evaluation against a road test in HD.

\section{METHOD}

\section{Participants}

Thirty persons with HD, recruited through the Movement Disorders Clinic of the University Hospitals Leuven, gave informed consent. Diagnosis of manifest HD was confirmed by DNA analysis and presence of clinical signs of chorea. Eligibility criteria were (1) valid driver's license; (2) driving for at least $300 \mathrm{~km}$ in the year prior to assessment; (3) binocular visual acuity scores of 20/40 or higher; and (4) no motor, visual, or psychiatric conditions unrelated to the disease. All procedures were approved by the local Ethics Committee.

\section{Procedure}

The road test and driving simulator evaluation took place in random order at two locations (Brussels and Leuven). The median (Q1 - Q3) interval between test moments was 7 (2 - 24) days. The on-road and driving simulator assessor were blind to each other's scores. 
On-road drive. The 20-km standardized road test was performed between 9 AM and 4 PM in the vicinity of Brussels using an Opel Astra fitted with dual controls to ensure driving safety. First, participants were instructed to adjust the driver's seat and mirrors. To familiarize themselves with the steering wheel and pedals, participants drove two laps on a closed course. The actual onroad test started in a suburban area with low to moderate traffic, progressed to a 6-lane, 2-way highway, continued to an urban area, and terminated at the driving evaluation center.

Driving simulation. The driving simulator is a full-sized Ford fiesta 1.7 with all its original mechanical parts. For standardization, automatic gear transmission was used for all participants. The simulator scenarios ran on STISIM software, version 2.10.05 (STI Inc., Hawthorne, CA). The interactive scenarios were projected on a flat screen with dimensions of approximately $2.30 \mathrm{~m}$ by $1.70 \mathrm{~m}$. An audio system generated synchronized natural sounds, including the sound of the engine for enhanced realism. Prior to the evaluation scenario, participants got familiarized with the driving simulator hardware and software by driving four 2-km practice scenarios with increasing complexity. The fourth practice scenario was similar to the actual evaluation, which was about $15 \mathrm{~km}$ long. The simulator evaluation started on suburban roads with low traffic density (speed limit $70-90 \mathrm{~km} / \mathrm{h}$ ), proceeded from low to high traffic urban areas (speed limit $50 \mathrm{~km} / \mathrm{h}$ ), continued to a 2-way, 4-lane highway road with low to heavy traffic, and ended in an urban area with high traffic density. Also, unexpected hazardous events were simulated such as road blocks due to in-lane traffic accidents, children crossing the road, and an approaching vehicle drifting into the driver's lane that required the driver to make an emergency brake and/or to perform an evasive maneuver. Computer-generated scores were collected including number of traffic violations, crashes, and divided attention (DA) measures. Drivers were instructed to respond to 28 symbols depicting horns or turn signals by honking the horn or using the turn signal indicators, respectively (Devos et al., 2012). Participants were advised to report any simulator discomfort to the assessor.

\section{Test Ride for Investigating Practical fitness to drive (TRIP)}

The TRIP checklist was developed at the University of Groningen, The Netherlands (Withaar, 2000). Content validity was established after revision of the TRIP by driving instructors from Dutch and Belgian fitness to drive evaluation centers. We used a modified version as some items were found to be unreliable in assessing driving ability after stroke (Akinwuntan et al., 2003).

On-road TRIP (Table 1). The on-road TRIP contains 13 items and 49 subitems on a 4-point ordinal scale (poor, insufficient, sufficient, and good) using clear-cut guidelines for each of the 49 subitems. The 13 items of the TRIP are closely linked to the hierarchical structure of the driving task (Michon, 1989). The operational factor consisted of items 1 to 3 . The tactical factor comprised the items 4 to 8 . Higher-order visual skills included items 9 to 11. Finally, a mixed factor involved the items 12 and 13. A pass-fail decision was based on the participants' performance on the TRIP test. A pass decision meant that drivers showed no functional deficits that interfered with driving or exhibited adequate strategies to compensate for their loss of functioning. Drivers who demonstrated poor driving skills or required repeated verbal or physical intervention were assigned to the fail category. 
Driving simulator TRIP (Table 1). The on-road TRIP checklist was adapted for the driving simulator evaluation since some of the on-road events were not part of the driving simulation (e.g., joining the traffic stream) and some sub-items of the on-road TRIP checklist could not be evaluated in our driving simulator (e.g., checking blind spot or side view mirrors).

Table 1. Score ranges for on-road TRIP items and driving simulator TRIP items

\begin{tabular}{llll}
\hline Item & Variable & On-road & Driving simulator \\
\hline & Total score & $49-196$ & $30-120$ \\
1 & Lateral position on the road at speed below $50 \mathrm{~km} / \mathrm{h}$ & $2-8$ & $2-8$ \\
2 & Lateral position on the road at speed above $50 \mathrm{~km} / \mathrm{h}$ & $2-8$ & $1-4$ \\
3 & Mechanical operations & $3-12$ & $3-12$ \\
4 & Speed adaptations at speed below $50 \mathrm{~km} / \mathrm{h}$ & $2-8$ & $1-4$ \\
5 & Speed adaptations at speed above $50 \mathrm{~km} / \mathrm{h}$ & $2-8$ & $2-8$ \\
6 & Gap distance at speed below $50 \mathrm{~km} / \mathrm{h}$ & $2-8$ & $2-8$ \\
7 & Gap distance at speed above $50 \mathrm{~km} / \mathrm{h}$ & $2-8$ & $3-12$ \\
8 & Lane position change & $5-20$ & $4-16$ \\
9 & Anticipation and perception of traffic light and signs & $4-16$ & $1-4$ \\
10 & Visual behavior and communication & $8-32$ & $2-8$ \\
11 & Understanding, insight and quality of traffic participation & $2-8$ & $7-28$ \\
12 & Turning left & $9-36$ & $6-24$ \\
13 & Joining the traffic stream* & & \\
\hline
\end{tabular}

* Item was not assessed in the driving simulator.

\section{Data analysis}

One participant could not be tested in the simulator due to technical issues, leaving 29 subjects in the analysis. Participants' demographic and driving data were described using means \pm standard deviation, median (Q1 - Q3), or frequencies (\%). Spearman rho coefficients were used correlate the driving simulator TRIP items with the on-road scores. Correlations were considered weak below 0.10, moderate between 0.10 and 0.49, and strong between 0.50 and 1.00 (Cohen, 1992). A logistic regression analysis was employed to calculate the variance of on-road decisions predicted by the total simulator score.

\section{RESULTS}

Descriptive and driving characteristics are displayed in Table 2. Disease characteristics can be found in a previous study (Devos et al., 2012).

Table 2. Descriptive and driving characteristics of the HD group $(n=29)$

\begin{tabular}{lc}
\hline Age, y & $50 \pm 13$ \\
Gender, male (\%) & $21(72)$ \\
Education, y & $14 \pm 4$ \\
Driving experience, y & $30 \pm 12$ \\
Annual mileage, $10^{3} \mathrm{~km} / \mathrm{y}$ & $10(5-15)$ \\
\hline
\end{tabular}

Abbreviation: HD, Huntington disease.

Results are presented as means \pm standard deviation, medians (Q1 - Q3) or frequencies (\%).

The computer-generated measures of the simulator data correlated strongly with the on-road TRIP scores, except for the number of traffic violations (Table 3). 
Table 3. Computerized simulator output and correlation with total on-road TRIP in 29 drivers with HD

\begin{tabular}{llcc}
\hline Simulator output & Median $(\mathrm{Q} 1-\mathrm{Q} 3)$ & $\mathrm{r}_{\mathrm{S}}$ & $\mathrm{P}$ value \\
\hline Traffic violations & $1(1-2)$ & $0.06^{*}$ & 0.76 \\
Crashes & $2(1-3)$ & $-0.58^{* * *}$ & 0.001 \\
DA response time & $16(12-19)$ & $-0.56^{* * *}$ & 0.002 \\
DA correct answers & $3.37(2.04-5.34)$ & $0.56^{* * *}$ & 0.002 \\
\hline
\end{tabular}

Abbreviations: DA, divided attention; HD, Huntington disease; $r_{s}$, spearman rho correlation coefficient; TRIP, Test Ride for Investigating Practical fitness to drive.

* weak correlation; ** moderate correlation; *** strong correlation.

One participant reported mild signs of the simulator adaptation syndrome (SAS) but chose to complete the simulator drive. There was a ceiling effect of both the driving simulator and onroad TRIP items (Table 4). Correlation coefficients ranged from 0.12 (item 6) to 0.72 (total TRIP score).

Table 4. Performance and correlations of the driving simulator and on-road TRIP in 29 drivers with HD

\begin{tabular}{|c|c|c|c|c|c|}
\hline Item & Variable & $\begin{array}{l}\text { Driving simulator } \\
\text { Median (Q1 - Q3) }\end{array}$ & $\begin{array}{c}\text { On-road } \\
\text { Median (Q1 - Q3) }\end{array}$ & $\mathrm{r}_{\mathrm{s}}$ & $\mathrm{P}$ value \\
\hline & Total score & $113(89-120)$ & $187(151-196)$ & $0.72 * * *$ & $<0.0001$ \\
\hline 1 & Lateral position on the road at speed below $50 \mathrm{~km} / \mathrm{h}$ & $8(6-8)$ & $8(6-8)$ & $0.62 * * *$ & 0.0004 \\
\hline 2 & Lateral position on the road at speed above $50 \mathrm{~km} / \mathrm{h}$ & $3(3-4)$ & $8(6-8)$ & $0.59 * * *$ & 0.0008 \\
\hline 3 & Mechanical operations & $12(9-12)$ & $11(8-12)$ & $0.67 * * *$ & $<0.0001$ \\
\hline 4 & Speed adaptations at speed below $50 \mathrm{~km} / \mathrm{h}$ & $4(3-4)$ & $7(6-8)$ & $0.38 * *$ & 0.06 \\
\hline 5 & Speed adaptations at speed above $50 \mathrm{~km} / \mathrm{h}$ & $7(6-8)$ & $7(6-8)$ & $0.50 * * *$ & 0.0006 \\
\hline 6 & Gap distance at speed below $50 \mathrm{~km} / \mathrm{h}$ & $8(8-8)$ & $8(6-8)$ & $0.12 *$ & 0.53 \\
\hline 7 & Gap distance at speed above $50 \mathrm{~km} / \mathrm{h}$ & $8(8-8)$ & $8(6-8)$ & $0.32 * *$ & 0.0008 \\
\hline 8 & Lane position change & $12(10-12)$ & $20(18-20)$ & $0.47 * *$ & 0.0009 \\
\hline 9 & Anticipation and perception of traffic light and signs & $15(12-16)$ & $16(12-16)$ & $0.32 * *$ & 0.009 \\
\hline 10 & Visual behavior and communication & $28(24-28)$ & $36(33-36)$ & $0.36 * *$ & 0.06 \\
\hline & Understanding, insight and quality of traffic participation & $8(6-8)$ & $8(6-8)$ & $0.49 * *$ & 0.002 \\
\hline 12 & Turning left & $4(3-4)$ & $31(24-32)$ & $0.55^{* * *}$ & 0.007 \\
\hline 13 & Joining the traffic stream $\dagger$ & NA & $24(20-24)$ & & \\
\hline
\end{tabular}

Abbreviation: HD, Huntington disease; NA, not applicable; TRIP, Test Ride for Investigating Practical fitness to drive.

* weak correlation; ** moderate correlation; *** strong correlation.

†Item was not assessed in the simulator.

Total scores on the simulator TRIP explained 32\% of the on-road decisions (odds ratio = 0.91; 95\% confidence interval $(0.84-0.98) ; \mathrm{p}=0.01)$ and correctly classified 23 patients (Table 5).

Table 5. Predictive accuracy of driving simulation

\begin{tabular}{|c|c|c|c|}
\hline \multirow[b]{2}{*}{ Simulation TRIP } & \multicolumn{2}{|c|}{ Road TRIP } & \multirow[b]{2}{*}{ Total } \\
\hline & Fail & Pass & \\
\hline Fail & 8 (a) & 1 (b) & 9 \\
\hline Pass & 5 (c) & $15(d)$ & 20 \\
\hline Total & 13 & 16 & 29 \\
\hline
\end{tabular}

\section{DISCUSSION}

The aim of this study was to investigate the concurrent validity of a driving simulation against a road test in active drivers with manifest HD. We found a strong correlation between the total driving simulator and on-road TRIP scores, suggesting that the comprehensive driving simulator evaluation provides a valid index of overall on-road driving performance. The predictive 
accuracy of the driving simulation (79\%) was lower than the predictive accuracy of a clinical screening battery (87\%) that we developed in a previous study (Devos et al., 2012). The modest sensitivity and NPV suggest that drivers with HD who are predicted to pass are referred for onroad testing as clinically appropriate. The specificity of the driving simulator evaluation was higher than that of the clinical screening battery (94\% vs $87 \%$ ), confirming previous findings in PD that driving simulators may be used complementary to clinical screening batteries for predicting persons who are likely to fail (=PPV) a driving evaluation (Devos et al., 2007). However, even in simulator failure, referral for road testing may still be needed when the clinical impression suggests otherwise or when there is resistance from the patient to accept the results.

We found significant correlations for 9 of the 12 items. Items assessing vehicle control (lateral position on the road, mechanical operations) were all highly correlated, suggesting that the driving simulation validly reproduces operational on-road skills. Weak to moderate correlations were found for tactical items. The items speed adaptation and gap distance below $50 \mathrm{~km} / \mathrm{h}$ were not significant. We observed that some drivers were having difficulties adhering to the speed limit in urban areas, possibly because of the lack of a movement base platform. Others may have felt overwhelmed by the constant flow of information of the urban events and drove slower than in real-life. Participants also kept a longer distance to the lead vehicle than they would in reallife, probably because of problems with depth perception due to the projections of images onto a single flat screen. Visual behavior and communication, an item that requires higher order visual processing, was also found not significant. Checking the side mirrors and blind spot could not be assessed in the driving simulator because of the single screen, which may be the reason for the non-significant finding. Using a more advanced driving simulator with a wide field of view may be more suited to investigate whether concurrent validity can also be established for speed adaptations and gap distance in urban areas, and visual behavior and communication.

Inherent to naturalistic driving, there were unexpected events in the road test (e.g. lead car making a sudden stop; not giving right-of-way of other drivers, speed bumps in the road), which were not featured in the simulator. Conversely, hazardous events and DA tasks were created in the simulator that did not occur during the on-road evaluation. The absence of validated SAS measures may have masked any symptoms of simulator discomfort and poses another limitation.

In conclusion, the present study validated a comprehensive driving simulator evaluation by using the same criteria as an official on-road evaluation. Our results support the concurrent validity of the driving simulator, especially for skills requiring vehicle control. While this study concentrated on drivers with HD, our results suggest that driving simulation is also valid to evaluate driving fitness in other populations with motor and cognitive dysfunction.

\section{ACKNOWLEDGMENTS}

The authors thank the CARA staff for the on-road driving evaluations. H.D. is a postdoctoral fellow and W.V. is a Senior Clinical Investigator of the Research Foundation - Flanders.

\section{REFERENCES}

Akinwuntan, A.E., Wachtel, J., and Newman Rose, P. (2012). Driving simulation for evaluation and rehabilitation of driving after stroke. Journal of Stroke and Cerebrovascular Diseases, 21(6), 478-486. 
Akinwuntan, A.E., De Weerdt, W., Feys, H., Baten, G., Arno, P., and Kiekens, C. (2003). Reliability of a road test after stroke. Archives of Physical Medicine and Rehabilitation, 84(12), 1792-1796.

Beglinger, L.J., Prest, L., Mills, J.A., Paulsen, J.S., Smith, M.M., Gonzalez-Alegre, P., Rowe, K.C., Nopoulos, P., and Uc, E.Y. (2012). Clinical predictors of driving status in Huntington disease. Movement Disorders, 27(9), 1146-1152.

Cohen, J. (1992). "A power primer". Psychological Bulletin, 112 (1), 155-159.

Devos, H., Nieuwboer, A., Tant, M., De Weerdt, W., and Vandenberghe, W. (2012). Determinants of fitness to drive in Huntington disease. Neurology, 79(17), 1975-1982.

Devos, H., Akinwuntan, A.E., Nieuwboer, A., Tant, M., and De Weerdt, W. (2011). Screening for fitness to drive after stroke: a systematic review and meta-analysis. Neurology, 76(8), 747-756.

Devos, H., Vandenberghe, W., Nieuwboer, A., Tant, M., and De Weerdt, W. (2007). Predictors of fitness to drive in people with Parkinson disease. Neurology, 69(14), 1434-41.

Fox, G.K., Bowden, S.C., and Smith, D.S. (1998). On-road assessment of driving competence after brain impairment: review of current practice and recommendation for a standardized examination. Archives of Physical Medicine and Rehabilitation, 79(10), 1288-1296.

Lee, H. C., Falkmer, T., Rosenwax, L., Cordell, R., Granger, A., and Lee, A. H. (2007). Validity of driving simulator in assessing drivers with Parkinson's Disease. Advances in Transportation Studies: an International Journal, Special Issue, 81-90.

Lundqvist, A., Gerdle, B., and Rönnberg, J. (2000). Neuropsychological aspects of driving after a stroke - in the simulator and on the road. Applied Cognitive Psychology, 14, 135-150.

Mayhew, D.R., Simpson, H.M., Wood, K.M., Lonero, L., Clinton, K.M., and Johnson, A.G. (2011). On-road and simulated driving: concurrent and discriminant validation. Journal of Safety Research, 42(4), 267-275.

Michon, J.A. (1989). Explanatory pitfalls and rule-based driver models. Accident Analysis and Prevention, 21(4), 341-353.

Nouri, F.M., and Tinson, D.J. (1988). A comparison between a driving simulator and a road test in the assessment of driving ability after stroke. Clinical Rehabilitation, 2, 99-104.

Patomella, A., and Kottorp, A. (2005). An evaluation of driving ability in a simulator. A good predictor of driving ability after stroke? Proceedings of the Third International Driving Symposium on Human Factors in Driver Assessment, Training and Vehicle Design, Rockport, Maine, 104-110.

Uc, E.Y., Rizzo, M., Johnson, A.M., Emerson, J.L., Liu, D., Mills, E.D., Anderson, S.W., and Dawson, J.D. (2011). Real-life driving outcomes in Parkinson disease. Neurology, 76(22), 1894-1902.

Withaar, F.K. (2000). Divided attention and driving: The effects of aging and brain injury. PhD Thesis. University of Groningen, The Netherlands. 